\title{
Association Between Acute Stroke and Metabolic Syndrome
}

\author{
MD. J ABEDIQBAL, ${ }^{1}$ MD. MAHMUDUR RAHMAN SIDDI QUI , ${ }^{2}$ QUAZ TARI KUL ISLAM, ${ }^{3}$ AHMED HOSSAI N, ${ }^{4}$ EKRAMUL \\ MUSTAFA, ${ }^{5}$ MD. RUHUL AMI N SHIPA, ${ }^{2}$ MD. YOUSUF UR RAHMAN, ${ }^{6}$ HASHMI SI NA ${ }^{7}$
}

\begin{abstract}
Objective: Metabolic syndrome is the cluster of vascular risk factors including insulin resistance, elevated blood pressure, hyperlipidemia, and obesity. Metabolic syndrome is strongly associated with cardiovascular and cerebrovascular disease. The aim of this study is to find out association between stroke and metabolic syndrome in our population.
\end{abstract}

Methods: In this observational study, a total number of fifty randomly selected patients were studied from July 2009 to December 2009 at Medicine ward of Dhaka Medical College Hospital. Definition of metabolic syndrome was taken from the guidelines by the National Cholesterol Education Program Adult Treatment Panel III.

Results: Among the study population $46 \%$ had metabolic syndrome. This study estimated that Ischaemic stroke is more common among metabolic syndrome patients (65.2\%), but patient without metabolic syndrome had more haemorrhagic stroke (55.6\%).Only I $2 \%$ patient was found obese. With or without metabolic syndrome most of the patient had elevated TG (64\%) and raised blood pressure (64\%).

Conclusion: Without obesity, metabolic syndrome is an important risk factor for cerebrovascular disease. Majority of the patients with metabolic syndrome present as ischemic stroke.

Keyword: Stroke, Metabolic syndrome, hypertension, fasting blood sugar, hyperlipidaemia.

\section{Introduction}

Stroke is the most common neurological emergency; the third most common cause of death in developed world. 85\% strokes are ischemic, $10 \%$ are intracranial hemorrhages (ICH), and $5 \%$ are subarachnoid hemorrhages (SAH). ${ }^{1}$ WHO defines stroke as a clinical syndrome characterized by rapidly developing clinical symptoms and/or signs of focal loss of cerebral function with symptoms lasting more than 24 hours or leading to death before that with no apparent cause other than that of vascular origin. ${ }^{1}$ Metabolic syndrome is associated with an increased risk of cardiovascular events and ischemic stroke. ${ }^{2}$ One study showed that patient with metabolic syndrome had a 1.6 fold increased risk of stroke. ${ }^{2}$ Metabolic syndrome is the cluster of vascular risk factors including insulin resistance, elevated blood pressure, hyperlipidemia, and obesity. According to The National Cholesterol Education Program Adult Treatment Panel III (ATP-III), definition of metabolic syndrome requires the presence of 3 or more of the following criteria: abdominal obesity (waist circumference e"102cm in men and e" $88 \mathrm{~cm}$ in women) for Asian, IDF criteria is e" $90 \mathrm{~cm}$ in men and e" $80 \mathrm{~cm}$ in women. The ATP III update accepted these same criteria. A high triglyceride level ( $>150 \mathrm{mg} / \mathrm{dl}$ or $>1.69 \mathrm{mmol} / \mathrm{L}$ ), a low HDL cholesterol level $(<40 \mathrm{mg} / \mathrm{dl}$ or $<1.03 \mathrm{mmol} / \mathrm{L}$ for men and $<50 \mathrm{mg} / \mathrm{dl}$ or $<1.29 \mathrm{mmol} / \mathrm{L}$ for women), high blood pressure (Systolic $>130 \mathrm{~mm} \mathrm{Hg}$ or diastolic $>85 \mathrm{~mm} \mathrm{Hg}$ ) and a high fasting plasma glucose concentration $(>100 \mathrm{mg} / \mathrm{dl}) .{ }^{3,4}$ Metabolic syndrome increases the morbidity and mortality of cardiovascular diseases. ${ }^{5,6}$ However, few studies have examined the association between the incidence of stroke and metabolic syndrome. The aim of this study was to identify the association between stroke and metabolic syndrome patients admitted in a tertiary care hospital, as defined by criteria ATP III.

\section{Materials \& Methods}

It was a prospective observational type of study. The study was carried out in inpatient department of medicine, Dhaka

1. Postgraduate resident, Dept. of Medicine, Dhaka Medical College Hospital \& consultant cardiologist, The Medical Center, Dhanmondi, Dhaka.

2. Postgraduate resident, Dept. of Medicine, Dhaka Medical College Hospital.

3. Professor, Dept. of Medicine, Dhaka Medical College Hospital.

4. Assistant Professor, Dept. of Medicine, Dhaka Medical College Hospital.

5. Indoor Medical Officer, Dept. of Medicine, Dhaka Medical College Hospital.

6. Assistant Registrar, Dept. of Medicine, Dhaka Medical College Hospital.

7. Registrar, Dept. of Medicine, Dhaka Medical College Hospital.

Correspondence: Dr. Md. Jabed Iqbal, Postgraduate resident, Dept. of Medicine, Dhaka Medical College Hospital \& consultant cardiologist, The Medical Center, Dhanmondi, Dhaka. E-mail: dr.jabed.iqbal@gmail.com 
medical college Hospital, Dhaka, Bangladesh for a period of 6 months (July 2009-December 2009). This study was performed among the patients who were admitted in Medicine department of Dhaka Medical College Hospital, with clinical features and CT scan findings of strokes. A total of 50 patients' were enrolled in the study. Clinically suspected stroke patients were admitted. CT scan was done within 24 hours of hospital admission. Serum fasting lipid profile and fasting glucose level were determined within 24 hours after admission. Patient admitted after 48 hours of onset of symptoms, neurological deficit due to other than stroke like head injury, metabolic coma, trauma, poisoning or epilepsy and CT scan showing findings other than infarct or hemorrhage were excluded from this study. Metabolic syndrome was diagnosed as per ATP-III criteria. On admission, fasting lipid profile and Fasting blood sugar was done in next morning. All patients/legal guardians were informed about the study. Detailed history, clinical examination and relevant investigation reports of all patients were recorded in pre designed data collection sheet. Statistical analysis was conducted on SPSS 16.0 for windows software.

\section{Results}

Maximum patients (30\%) were in between the group of 51-60 years age and next prevalence group was $61-70$ years (22\%) ages. Out of 50 patients 27 ischaemic \& 23 were haemorrhagic stroke. 32 were male $\& 18$ were female patients.

Fig 1 showing, HDL level was low in 68\% of patients, among them ischaemic stroke patients had much low (70.4\%) level of HDL then haemorrhagic stroke.

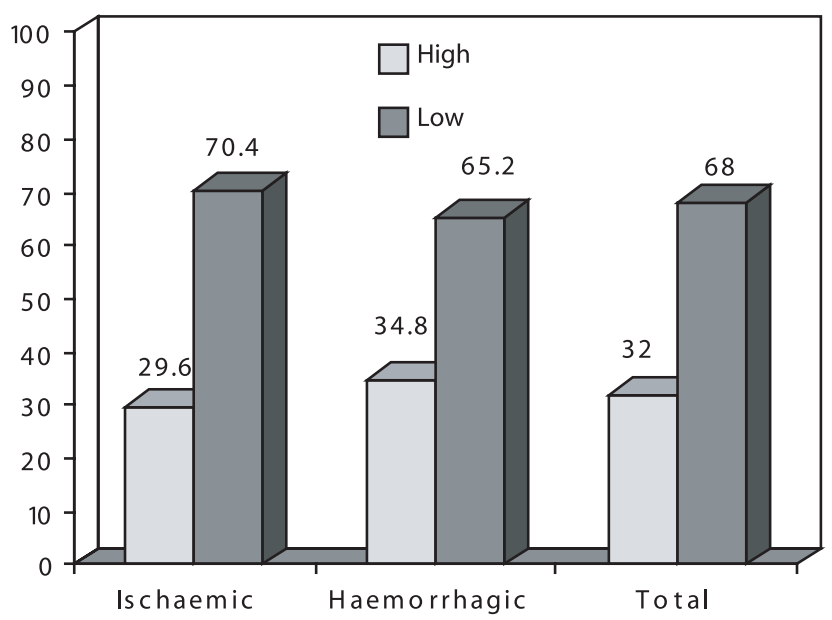

Fig.-1: Level of high density lipoprotein (HDL) among various type of stroke $(n=50)$.
Fig 2 showing, Triglyceride level was high in $64 \%$ of stroke patients, among them ischaemic stroke patients had more high level of $\operatorname{Tg}$ (70.4\%) then haemorrhagic patients.

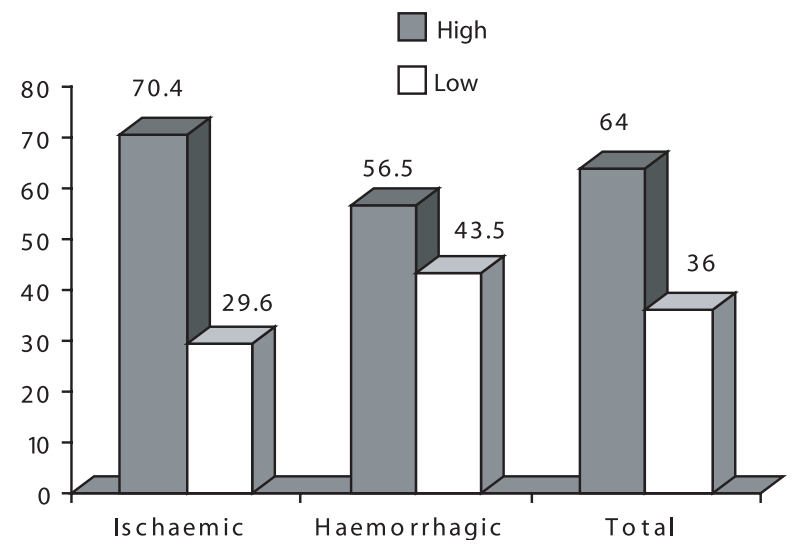

Fig.-2: Level of Triglyceride (Tg) among various type of stroke $(n=50)$.

Fig 3 showings, 44\% of stroke patients have high FBS. Ischaemic stroke patients had more high FBS level (48.1\%).

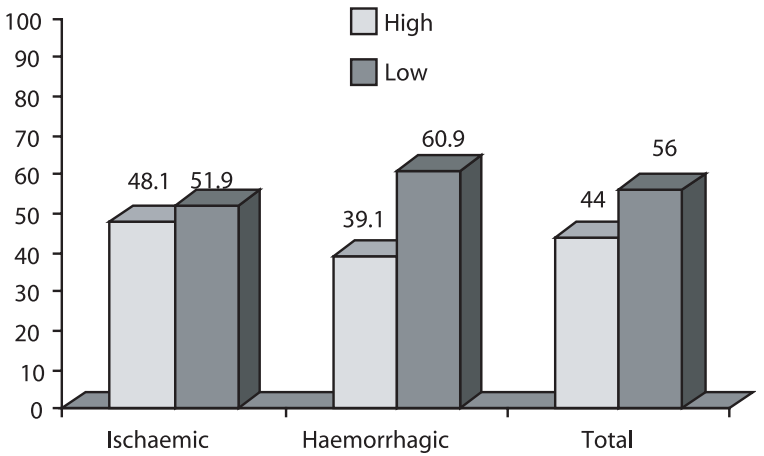

Fig.-3: Level of fasting blood sugar (FBS) among different type of stroke $(n=50)$.

Fig 4 showing, only $12 \%$ stroke patients have high waist circumference \& more in ischaemic stroke (19.5\%).

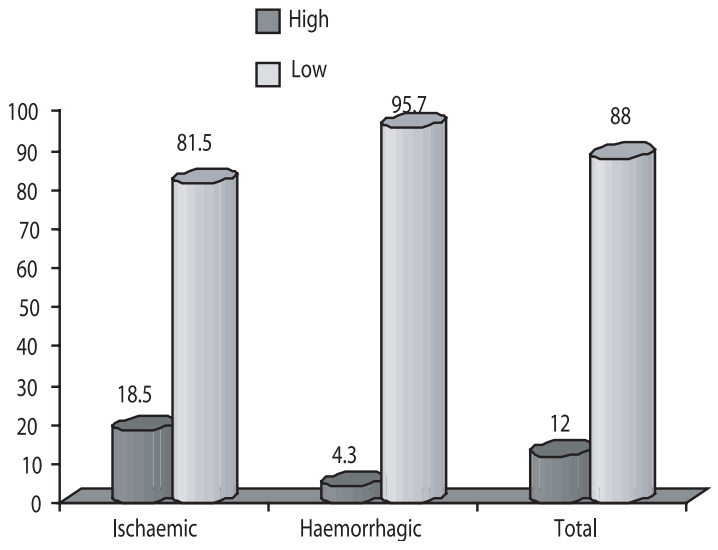

Fig-4: Waist circumference in different strokes $(n=50)$. 
Fig 5 showing, 64\% of patients had elevated blood pressure. $69.6 \%$ of haemorrhagic stroke $\& 59.3 \%$ of ischaemic stroke patients have elevated blood pressure.

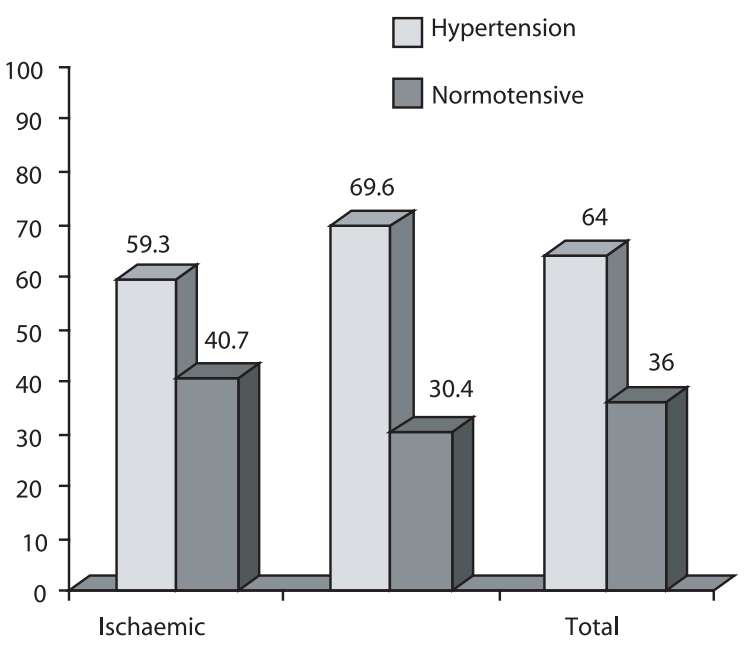

Fig.-5: Elevated blood pressure in various stroke $(n=50)$.

Fig 6 showing 46\% of all stroke patients have metabolic syndrome.

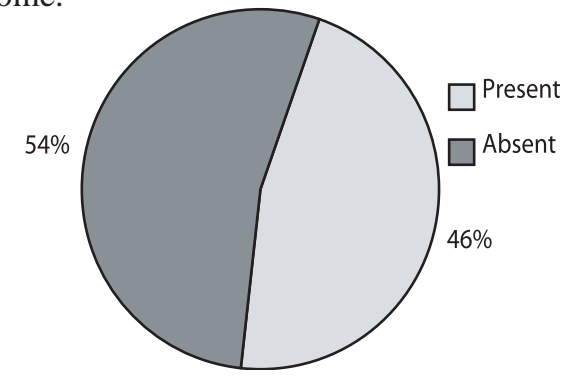

Fig-6: Metabolic syndrome among all stroke patients $(n=50)$.

Fig 7 showing, ischaemic stroke is more common among metabolic syndrome patients (65.2\%), but patient without metabolic syndrome had more haemorrhagic stroke (55.6\%).

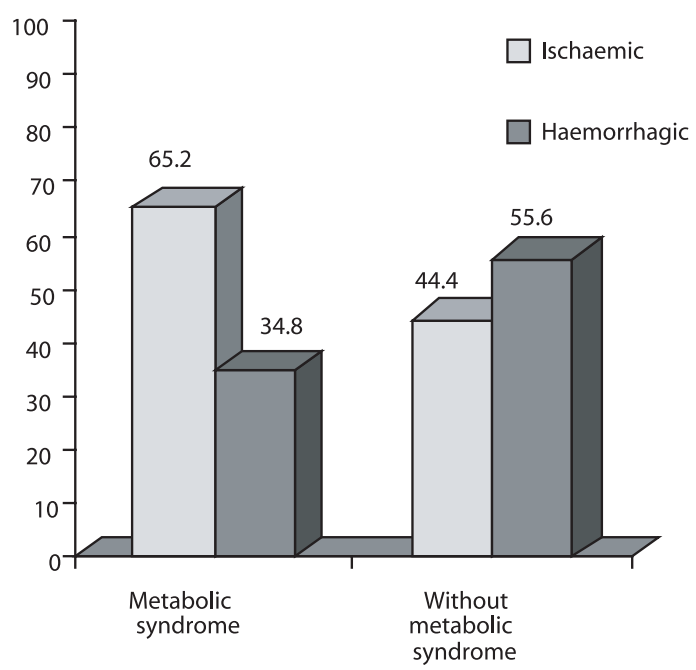

Fig-7: Metabolic syndrome in different types of stroke $(n=50)$

\section{Discussion}

The prevalence of the metabolic syndrome (MetS) is increasing to epidemic proportions not only in the United States and the remainder of the urbanized world but also in developing nations and an increasing trend has been observed in Asian countries. ${ }^{7,8}$ Urbanization, modern lifestyle, change of food habit cumulatively contributes for this development of metabolic syndrome. We have applied the NCEP ATP III panel criteria 2002 to assess the prevalence and pattern of metabolic syndrome among Bangladeshi stroke patients. This study was carried out in the admitted stroke patients at Dhaka Medical College Hospital to find out association between metabolic syndromes (MetS) with stroke patients admitted in a tertiary care hospital. Maximum patients (30\%) were in between the group of 51-60 years age and next prevalence group was 61-70 years (22\%) ages. Regarding the cause of stroke, as revealed by CT scan, majority had stroke due to infarction. Out of 50 patients 27 ischaemic \& 23 were haemorrhagic stroke. 32 were male \& 18 were female patients. About half (46\%) of all admitted stroke patients have metabolic syndrome. In a study in Mitford Hospital in the year 2008, they had found metabolic syndrome among admitted stroke patients was $48 \% .{ }^{9}$ This is very close to our study. Najarian and colleagues examined the risk for stroke in 2097 participants from the Framingham Offspring Study, of whom $22 \%$ had metabolic syndrome. ${ }^{10}$ But in our patients, prevalence of MetS in stroke patients was much higher (46\%). HDL level was low in 68\% of patients, among them ischaemic stroke patients had much low (70.4\%) level of HDL then haemorrhagic stroke. Triglyceride (Tg) level was high in $64 \%$ of stroke patients, among them ischaemic stroke patients had more high level of Tg (70.4\%) then haemorrhagic patients. 44\% of stroke patients had high FBS. Ischaemic stroke patients had more high FBS level (48.1\%). $10 \%$ of stroke patients had diabetes, frequency being same in both stroke. $64 \%$ of patients had elevated blood pressure. $69.6 \%$ of haemorrhagic stroke $\& 59.3 \%$ of ischaemic stroke patients have elevated blood pressure. Ischaemic stroke was more common among metabolic syndrome patients (65.2\%), but patient without metabolic syndrome had more haemorrhagic stroke (55.6\%). MetS is associated with higher risk of stroke and similar or higher risks then elevated fasting blood glucose alone or hypertension alone. ${ }^{11,12}$ Furthermore, there has been little prospective studies on the relationship between MetS and risk of stroke. ${ }^{12}$ Our findings suggest that metabolic syndrome was associated with an increased incidence of stroke. These result correlate with a study in Japan. ${ }^{13}$ In the Hisayama Study, metabolic syndrome, as defined by the modified ATP III criteria, was associated with increased morbidity for cardiovascular diseases, including 
stroke. ${ }^{14}$ In the NIPPON DATA 80, metabolic syndrome was associated with higher incidences of ischemic stroke. ${ }^{15}$ In our study $65.2 \%$ of ischaemic stroke patients had metabolic syndrome which was more than haemorrhagic stroke. In our study only $12 \%$ stroke patients had high waist circumference \& more in ischaemic stroke (19.5\%). In Japan they also found poor correlation of stroke with high waist circumference though high waist circumference is positively associated with the risk of cardiovascular events. ${ }^{13,16,17}$ The most notable limitation of this study is its small sample size. We believe that a longer period of follow up in a large population will solve the problem of small sample size.

\section{Conclusion}

In conclusion, metabolic syndrome, as defined by the ATP III criteria, was positively associated with stroke. Furthermore, in ischaemic stroke, there was a significant difference in stroke incidence between with and without metabolic syndrome. More than $50 \%$ of ischemic stroke patients are suffering from metabolic syndrome. Diagnosis and proper management of metabolic syndrome can be an important part of prevention of stroke. Further large population based study may give us real picture in our society.

\section{Conflict of interest: None}

\section{References:}

1. WHO STEPS Stroke Manual: the WHO STEP wise approach to stroke surveillance. STEPS Stroke Surveillance Manual (V2.1); 2006-05-09.

2. Li W, Ma D, Liu M, et al. Association between Metabolic syndrome and Risk of Stroke: A Meta-Analysis of Cohort Studies. Cerebrovasc dis 2008;25(6):539-47.

3. Park Y, Zhu S, Palaniappan L et al. The Metabolic Syndrome: Prevalence and Associated Risk Factor Findings in the US Population From the Third National Health and Nutrition Examination Survey, 1988-1994. Arch Intern Med 2003;163:427-436.

4. Grundy SM, Cleeman JI, Daniels SR, Donato KA, Eckel $\mathrm{RH}$, Franklin BA, et al for the American Heart Association; National Heart, Lung, and Blood Institute. Diagnosis and management of the metabolic syndrome: an American Heart Association/National Heart, Lung, and Blood Institute Scientific Statement. Circulation 2005; 12(17): 2735-2752.

5. Alberti KG, Zimmet PZ. Definition, diagnosis and classification of diabetes mellitus and its complications. Part
1: diagnosis and classification of diabetes mellitus provisional report of a WHO consultation. Diabet Med 1998;15:53953.

6. Reaven GM. Banting lecture 1988. Role of insulin resistance in human disease. Diabetes 1988; 37: 1595-607.

7. Al Lawati JA, Mohammed AJ, Al Hinai HQ, Jousilahti P: Prevalence of the metabolic syndrome among Omani adults. Diabetes Care 2003; 26:1781-1785.

8. Chen CH, Lin KC, Tsai ST, Chou P: Different association of hypertension and insulin-related metabolic syndrome between men and women in 8437 nondiabetic Chinese. Am J Hypertens 2000;13:846-853.

9. Kabir A, Sadeka MM, Ahmed MJ et al. Frequency of metabolic syndrome among stroke patients. J Medicine 2008; 9(1): 37-41.

10. Najarian RM et al. Metabolic syndrome compared with type 2 diabetes mellitus as a risk factor for stroke: The Framingham Offspring Study. Arch Intern Med 2006; 166:106-11.

11. Mozaffarian D, Kamineni A, Prineas RJ et al. Metabolic Syndrome and Mortality in Older Adults. Arch Intern Med. 2008;168(9):969-978.

12. Wannamethee SG, Shaper AG, Lennon L. Metabolic Syndrome vs Framingham Risk Score for Prediction of Coronary Heart Disease, Stroke, and Type 2 Diabetes Mellitus. Arch Intern Med 2005;165:2644-2650.

13. Niwa Y, Ishikawa S, Gotoh T et al. Association between stroke and metabolic Syndrome in a japanese population: Jichi medical school (jms) cohort study. J Epidemiol 2010;20(1):62-69.

14. Ninomiya T, Kubo M, Doi Y, et al. Impact of metabolic syndrome on the development of cardiovascular disease in a general Japanese population: the Hisayama study. Stroke 2007;38:2063-9.

15. Iso H, Sato S, Kitamura A, Imano H, Kiyama M, Yamagishi $\mathrm{K}$, et al. Metabolic syndrome and the risk of ischemic heart disease and stroke among Japanese men and women. Stroke 2007;38:1744-51.

16. de Koning L, Merchant AT, Pogue J, Anand SS. Waist circumference and waist-to-hip ratio as predictors of cardiovascular events: meta-regression analysis of prospective studies. Eur Heart J 2007;28:850-6.

17. Hu G, Tuomilehto J, Silventoinen K, Sarti C, Männistö S, Jousilahti P. Body mass index, waist circumference, and waisthip ratio on the risk of total and type-specific stroke. Arch Intern Med 2007;167:1420-7. 